\title{
Indirect searches for dark matter with AMS-02
}

\author{
P. Brun ${ }^{\mathrm{a}}$ \\ DSM/IRFU, Service de Physique des Particules, CEA/Saclay, 91191 Gif-sur-Yvette, France \\ Received: 30 January 2008 / Revised version: 13 May 2008 / \\ Published online: 27 June 2008 - (C) Springer-Verlag / Società Italiana di Fisica 2008
}

\begin{abstract}
AMS-02 is a multi-purpose spectrometer with superconducting magnet, and is designed for 3 years of data taking aboard the International Space Station. Its high performance regarding particle identification and energy measurement will allow performing indirect searches for dark matter (DM) in different channels simultaneously: gamma rays, positrons and antiprotons. AMS-02 sensitivity to those signals are presented and - provided the positron excess is due to DM signal - it is shown that it allows to probe new physics models in detail. Its high sensitivity could even be a unique opportunity to reach the Majorana nature of the DM particle through final state polarization effects.
\end{abstract}

PACS. 95.35.+d; 95.55. Vj

\section{Introduction}

Combinations of different cosmological probes indicate the presence in the Universe of non baryonic dark matter (DM), at the level of $84 \%$ of the total matter density. In an independent way, several models beyond the standard model of particle physics (with supersymmetry or extra spacetime dimensions) predict the existence of neutral, weakly-interacting massive particles [1]. Those could fill the Universe up to the observed matter density provided their co-moving density is regulated by primordial self-annihilations.

Nowadays, these annihilations could take place in the center of massive structures containing DM, like e.g. the center of our Galaxy or in Galactic clumps of DM. If efficient enough, annihilations of DM particles can become observable sources of primary cosmic rays. Due to a reduced standard background, anti-particles $\left(e^{+}, \bar{p}, \bar{D}\right)$ are amongst the most promising channels, together with $\gamma$ rays. In this article, we focus mainly on the charged channels.

\section{The AMS-02 space spectrometer}

AMS-02 is a particle physics detector designed for 3 years of data taking in the $\mathrm{GeV}-\mathrm{TeV}$ region [2]. The core of the instrument is a silicon tracker, surrounded by a superconducting magnet and veto counters. The spatial resolution is $10 \mu \mathrm{m}$ on 8 planes, and the value of the magnetic field is $B L^{2}=0.86 \mathrm{~T} \mathrm{~m}^{2}$. Two scintillator planes allow to measure

\footnotetext{
a email: pierre.brun@cea.fr
}

the time of flight with a $120 \mathrm{ps}$ precision and an imaging Cerenkov counter determines the charge and velocity within $\sim 0.1 \%$. Proton rejection is performed with a transition radiation detector and an electromagnetic calorimeter, each of them providing a $\sim 10^{2-3}$ level proton rejection. The calorimeter also measures the energy of electromagnetic particles with a few percent precision.

For what concerns DM channels $\left(e^{+}, \bar{p}, \bar{D}\right.$ and $\left.\gamma\right)$, the main challenge is the rejection of the important proton background. Table 1 summarizes the mean expected flux ratio for these channels with respect to proton background. Specific event selections, using all sub-detectors, allow the observation of those particles with a high level of background rejection. As a result of optimized event selections in Monte Carlo simulations, Table 1 shows the obtained acceptance ratios $-\mathcal{A}_{\text {channel }} / \mathcal{A}_{\text {proton }}$, that quantifies the capability of the detector to disentangle between the two types of particles. Also shown are the energy ranges for which background contamination is less than $1 \%[3-5]$. Although this paper does not present $\gamma$ channel in AMS-02, we emphasize the fact that its sensitivity to $\gamma$ signal is very high up to a few $\mathrm{TeV}[5,6]$, in particular with a specific trigger based on the calorimeter signals only [7].

\section{Derivation of DM indirect signals}

Apart from the misidentified particles, the other type of background to face when searching for dark matter is related to the conventional production of cosmic rays in the Galaxy. Those are presumably produced either in Supernovae (primaries, which are also accelerated) or by interaction in the interstellar medium (secondaries). In the case 
Table 1. Background rejections and sensitivity ranges

\begin{tabular}{lccc}
\hline & $e^{+}$ & $\bar{p}$ & $\gamma$ \\
\hline$\langle\Phi$ channel $/ \Phi$ protons $\rangle$ & $10^{3}$ & $10^{4}$ & $10^{3-5}$ \\
Mean acceptance ratio & $5.6 \times 10^{5}$ & $10^{6}$ & $10^{4-7}$ \\
Energy range $(\mathrm{GeV})$ & $1-300$ & $1-600$ & $2-2000$ \\
\hline
\end{tabular}

of $e^{+}$and $\bar{p}$, secondaries are dominant at the Earth in the energy range of interest $(1 \mathrm{GeV}$ to a few $\mathrm{TeV})$. If occurring, DM annihilations in the halo constitute an exotic production of primary anti-particles. The flux of DM-induced charged particles at the Earth $\Phi$ is driven by a transport equation of the form

$$
\partial_{z}\left(V_{\mathrm{c}} \Phi\right)-K \Delta \Phi+\partial_{E}\left\{b(E)-K_{\mathrm{EE}}(E) \partial_{E} \Phi\right\}=\mathcal{Q}(\mathbf{x}, E) .
$$

Here, $V_{\mathrm{c}}, K, b(E)$ and $K_{\mathrm{EE}}$ are parameters of the propagation model and respectively stand for the convective wind, the diffusion coefficient, the energy loss rate and the diffusive reacceleration term. $\mathcal{Q}$ is the source term and is discussed below. This equation is solved with cylindrical boundary conditions that correspond to the expected shape of the diffusion zone. Depending on the considered species, some terms can be neglected (like e.g. $b(E)$ for antiprotons) and different methods can be applied for the resolution $[8,9]$.

The source term, that contains the new physics and the modelling of the DM halo, can be expressed as

$$
\mathcal{Q}(\mathbf{x}, E)=\frac{1}{4 \pi} \frac{\langle\sigma v\rangle}{m_{\chi}^{2}} \frac{\mathrm{d} N}{\mathrm{~d} E}(E) \frac{\rho_{\chi}^{2}(\mathbf{x})}{\delta},
$$

where $\langle\sigma v\rangle$ is the thermally-averaged annihilation crosssection, $m_{\chi}$ is the DM particle mass, $\mathrm{d} N / \mathrm{d} E$ is the energy spectrum produced in the annihilation (which is discussed below) and $\rho_{\chi}$ is the DM density. $\delta$ is a statistical factor, equal to 2 for identical particles and 4 for a mixing of two populations (e.g. DM and anti-DM particles). Additional negative source terms can account for the disappearance of the particles by interaction in the Galactic disc.

Each pair annihilation of DM particles leads to the production of a pair of standard particles. The types and flavors of the produced particles are associated to a probability which is determined through the new physics model. As it is shown in the following, a possible determination of the final state mean $\mathrm{d} N / \mathrm{d} E$ spectrum can lead to the identification of the underlying theory.

It is important to notice that the presence of DM substructures could significantly enhance the annihilation rate. The density of those can either follow the overall DM density (clumps) or not, like e.g. in the case of minispikes around intermediate-mass black holes (IMBHs). Indeed, the rate being $\propto \rho^{2}$, the higher the variance of the DM distribution, the higher the exotic signal, as a clumpy distribution would lead to a boost $\propto\left\langle\rho^{2}\right\rangle /\langle\rho\rangle^{2}$ with respect to a smooth one. Although enhancement factors can be explicitly computed within given substructure scenar- ios $[9,10]$, we will here adjust the value of the enhancement (in fact, the distance at the nearest substructure) in order to reproduce the so-called positron excess [11-13]. In the following, the type of substructure will not be specified, but maybe IMBHs type should be preferred since the idea of sizeable clumpiness enhancement have recently been doubted [14].

\section{Prospects for AMS-02}

\subsection{Supersymmetry and extra-dimensions signals}

In this section, we consider two models for the DM particle, within supersymmetry (SUSY) and Kaluza-Klein (KK) scenarios with extra-dimensions. In the two cases, specific models and parameters are chosen to exhibit typical features of each scenario. In both cases, candidates of $150 \mathrm{GeV}$ mass are considered with sets of parameters that match cosmological constraints on the relic density. The computations of the exotic fluxes - including cross-sections, dark halo modelling and propagation - are performed with a new version of the micrOMEGAs package $[15,16]$.

In the minimal supersymmetric extension of the standard model (MSSM), the typical candidate is the lightest neutralino (LSP). The SUSY breaking scenario we consider here is a mSUGRA type with unification of the scalar masses to $m_{0}=113 \mathrm{GeV}$ and of the fermions to $m_{1 / 2}=$ $375 \mathrm{GeV}$ at an energy of $\mathcal{O}\left(10^{19}\right) \mathrm{GeV}$. We also fix $\operatorname{tg} \beta=50$, $\mu>0$ and a vanishing trilinear coupling. The mass spectrum is computed with the SuSpect package [17], and leads to a $150 \mathrm{GeV}$ neutralino. The bino component being dominant, the annihilation goes through a mixing of $b \bar{b}$ and $\tau^{+} \tau^{-}$pairs in a $60 / 40$ proportion. The total annihilation cross section, as computed with micrOMEGAs, is $\langle\sigma v\rangle=$ $7 \times 10^{-28} \mathrm{~cm}^{2} \mathrm{~s}^{-1}$.

The KK scenario used here considers warped extradimensions with a $\mathrm{SO}(10)$ unification group [18]. The DM particle in this scenario is referred to as the LZP (in reference to the name of the discrete symmetry that guarantees proton stability). In this framework, the annihilation occurs mainly via the exchange of $Z^{0}$ or $Z^{\prime}$ bosons in the $s$-channel. Considering KK bosons mass scale of $3 \mathrm{TeV}$, a proper thermal relic density can be obtained for a DM particle mass of $150 \mathrm{GeV}$ [18], with a total annihilation cross section $\langle\sigma v\rangle=2 \times 10^{-26} \mathrm{~cm}^{2} \mathrm{~s}^{-1}$. In this framework, $74 \%$ of the annihilation produce quark pairs, and the direct branching fraction into charged leptons is $3 \%$ each. This represents a high proportion of direct $e^{+} / e^{-}$pairs. The positrons which are produced this way are particularly interesting since they are mono-energetic.

Let us focus now on the specific features of each type of model. For what concerns antiprotons, they are exclusively produced in the quark hadronization processes ( $\tau$ channels do not produce $\bar{p}$ ). The quark yield being more important for the LZP, a little more $\bar{p}$ are expected in that case. However, the most important differences between the two spectrum predictions appear for positrons. Indeed, the relatively large production of $e^{+}$line in the LZP case lead to 

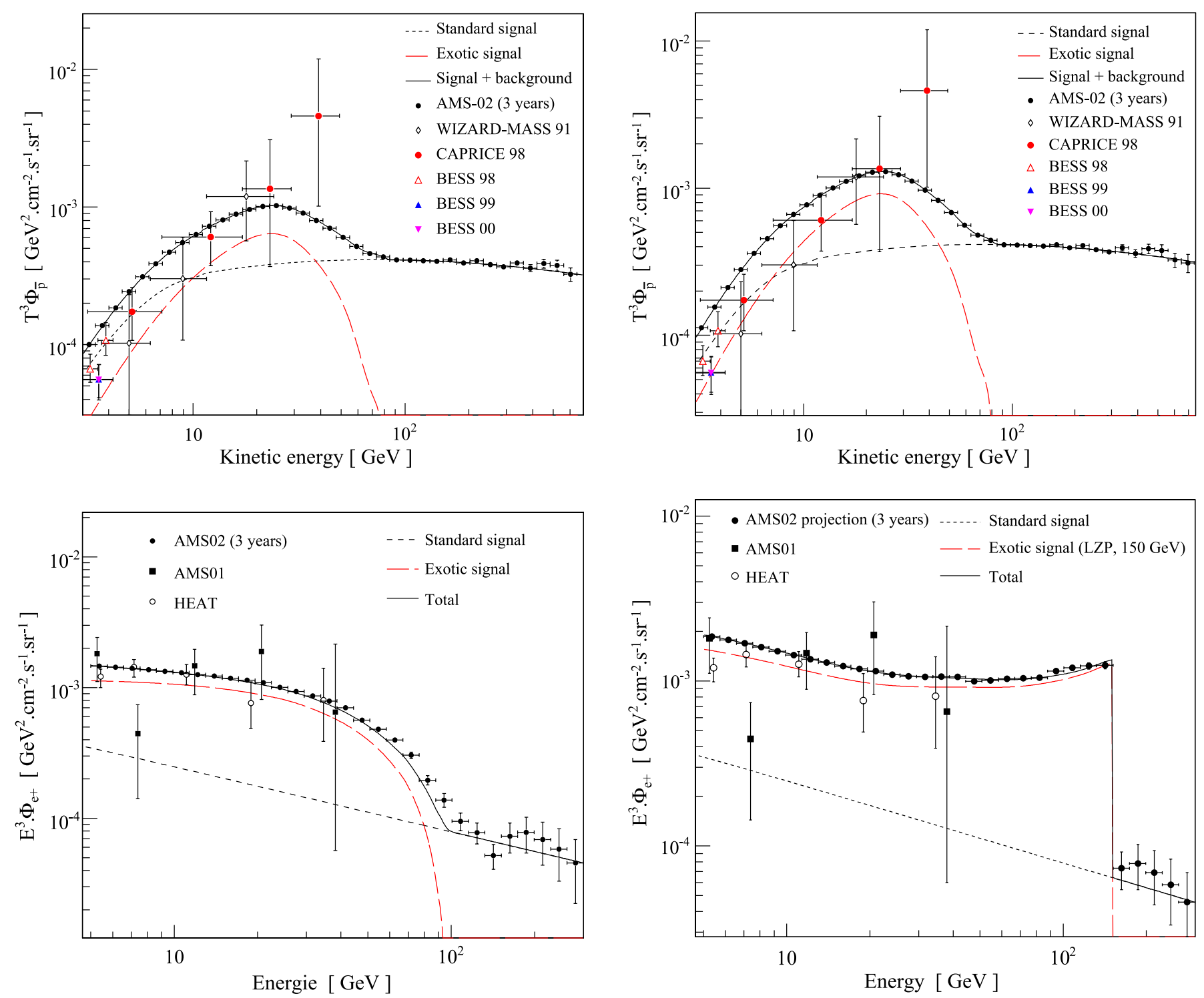

Fig. 1. Expected measurements of the $e^{+}$and $\bar{p}$ fluxes for SUSY DM: a bino (left) and a LZP (right)

a spectrum that exhibit a very sharp end at $150 \mathrm{GeV}$, which does not appear in SUSY. The scope of this paper is to show that such spectra can easily be identified with 3 years of data taking with AMS-02. To do so, we use a comprehensive simulation of the AMS-02 apparatus, and include all systematics related to misidentification of the different backgrounds. We include also statistical uncertainties accounting for 3 years of data taking. In the considered energy range, the theoretical uncertainties on the flux predictions decreadses as the energy increases. In fact, the higher the energy, the more purely diffusive the transport regime. As a consequence, high energy fluxes are more under control since they depend on less parameters. For that reason, fluxes are only considered above $3 \mathrm{GeV}$ here.

Figure 1 displays the different spectra as they would be measured by AMS-02, together with current measurements. The upper panels present $\bar{p}$ measurement and the lower panels show the $e^{+}$signals. In each case, the SUSY candidate is considered on the left and the KK one is on the right. Standard fluxes are determined from [19] for $\bar{p}^{1}$ and from [20] for $e^{+}$. One can see that, apart from the normalization of the signals, the $\bar{p}$ signals do not exhibit significant differences (this degeneracy in the $\bar{p}$ signal has been pointed at in [19]). On the contrary, as mentioned above, the $e^{+}$line production lead to significant dissimilarities in the positron spectra. It appears that the high sensitivity of AMS-02 to this channel allows to clearly distinguish the two models. In particular, the sharp cutoff in the KK-induced $e^{+}$spectrum can be detected with a high significance.

While we do not show the results for $\gamma$ rays expected measurements here, it is important to notice also that - un-

\footnotetext{
1 In the case presented here, the standard signal is consistent with BESS results and AMS-02 points are assumed to be slightly higher.
} 

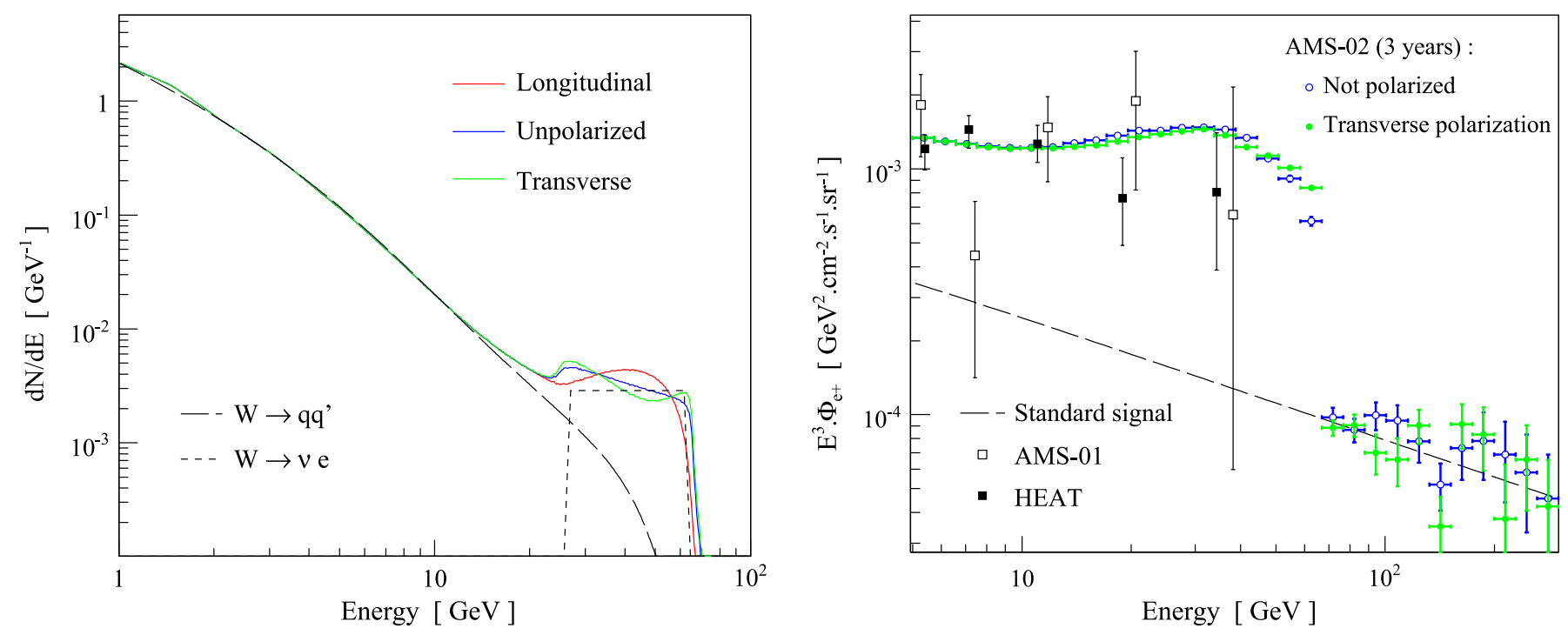

Fig. 2. $e^{+}$spectra for different polarization, before propagation (left) and as they would be measured by AMS-02 (right)

like the KK case - the SUSY case leads to an observable $150 \mathrm{GeV} \gamma$ line. This result is obtained thank to the high performance of the calorimeter.

Notice finally that, even for those relatively light neutralinos, fluxes measurements on a large energy range are essential. In fact, only a precise measurement of the spectra above the neutralino mass can lead to an identification of the expected spectral features. Furthermore, it allows a direct measurement of the standard secondary background and helps in fitting shape of the signal. From that point of view also, AMS-02 great advantage is to offer large energy range measurements.

\subsection{Polarization of final state gauge bosons}

In this section, we consider the specific case of an annihilation into pairs of gauge bosons $\left(\chi \chi \rightarrow W^{+} W^{-}\right)$. This occurs in the MSSM when the LSP has a large Higgsino component (like, e.g. in anomaly-mediated SUSY breaking scenarios), or within universal extra-dimensions frameworks.

We point out here that the energy spectrum of the outgoing $e^{+}$(and $\nu$ ) depends on the polarizations of the produced gauge bosons. Indeed, Majorana particles like SUSY neutralinos lead to transversally polarized gauge bosons, whereas Dirac particles such as KK candidates produce unpolarized bosons. $e^{+}$and $\nu$ spectra should be different in each case, as we shall see in the following. This effect has never been pointed at before, a more detailed study will appear in [21].

The left panel of Fig. 2 displays the $\mathrm{d} N / \mathrm{d} E$ functions for a generic $100 \mathrm{GeV}$ DM particle annihilating into $W$ pairs with different polarizations. The specific shapes of each curve are related to the angular distribution of the leptons in the $W \rightarrow \ell \nu$ processes, depending on the $W$ polarization. After propagation of these spectra, the differences between the two cases tend to vanish but we show that a sizeable difference stands when the fluxes are considered at the Earth.

In the same way as in previous section, the prediction for AMS-02 observations in 3 years of data taking are performed, assuming the DM signal is the origin of the positron excess. The right panel of Fig. 2 shows the expected AMS-02 measurements in each case. It appears that - under the assumption of a $W$ dominated annihilation (which could be inferred, e.g., from the LHC data) a few bins show significant differences, in particular around $20 \mathrm{GeV}$ and in the last bins of the excess. A precise analysis of the spectral shape of the excess could allow to distinguish between the two cases through a fit of the data points with hypotheses on the signal shape. Notice that the determination of the Majorana nature of the DM particle would be impossible with any current experiment, neither at the LHC. Only the future linear collider could reach that kind of information.

\section{Conclusions}

AMS-02 is a large acceptance space spectrometer that will perform precise measurements of cosmic radiation up to the $\mathrm{TeV}$ region, with a high level of background rejection. The prospect concerning indirect searches for dark matter are very promising in the antiprotons, positrons and gamma rays channels. In particular, it is shown that not only an excess can be observed, but specific new physics models can be unambiguously identified. The precise measurement of the high energy part (above the DM particle mass) insures to simultaneously have a determination of the standard background. In addition, the accurate determination of the nuclei fluxes will lead to important constraints on the propagation models and significantly reduce the theoretical uncertainties, thus allowing to reach even higher sensitivities to DM models. 
On top of that, the high sensitivity to positrons and the precision of the energy measurement allow to probe very fine details of the new physics underlying the dark matter problem. For example, if the positron excess is due to particles annihilating in gauge boson pairs, AMS-02 as enough sensitivity to give clues on the Majorana structure of the dark matter particle.

\section{References}

1. G. Bertone, D. Hooper, J. Silk, Phys. Rep. 405, 279 (2005)

2. http://ams.cern.ch

3. J. Pochon, Ph.D. Thesis LAPP-T-2005-05 (2005)

4. D. Caraffini, Ph.D. Thesis, AMS note 2005-07-02 (2004)

5. L. Girard, Ph.D. Thesis LAPP-T-2004-04 (2004)

6. A. Jacholkowska et al., Phys. Rev. D 74, 023518 (2006)

7. P. Brun, S. Rosier-Lees, astro-ph/0601537 (2006)
8. D. Maurin, C. Combet, R. Taillet, astro-ph/0609522 (2005)

9. J. Lavalle, J. Pochon, P. Salati, R. Taillet, Astron. Astrophys. 462, 827 (2006)

10. P. Brun, G. Bertone, J. Lavalle, P. Salati, R. Taillet, Phys. Rev. D 76, 083506 (2007)

11. S. Coutu et al., Astropart. Phys. 11, 429 (1999)

12. J.J. Beatty et al., Phys. Rev. Lett. 93, 241102 (2004)

13. M. Aguilar et al., Phys. Lett. B 646, 145 (2007)

14. J. Lavalle, Q. Yuan, D. Maurin, X.-J. Bi, arXiv:0709.3634 (2007)

15. P. Brun, astro-ph/0603387 (2006)

16. P. Brun, S. Rosier-Lees, G. Bélanger, F. Boudjema, A. Pukhov, in prep.

17. A. Djouadi, J.L. Kneur, G. Moultaka, hep-ph/0211331 (2002)

18. K. Agashe, G. Servant, Phys. Rev. Lett. 93, 231805 (2004)

19. T. Bringmann, P. Salati, Phys. Rev. D 75, 083006 (2007)

20. E.A. Baltz, J. Edsjö, Phys. Rev. D 59, 023511 (1999)

21. P. Brun, E. Nezri, J. Orloff, S. Rosier-Lees, in prep. 